Steve Kenner \& Dirk Lange

\title{
Bürgerbewusstsein, politisches Lernen und Partizipation im digitalen Zeitalter
}

\section{Zusammenfassung}

Ausgehend vom Stellenwert digitaler Medien und sozialer Netzwerke im Alltag junger Menschen werden in diesem Beitrag fachwissenschaftliche Zugänge aus den Citizenship Studies mit lerntheoretischen Überlegungen zum Bürgerbewusstsein verbunden. Hervorgehoben wird dabei das Verhältnis von digitaler Erfahrungs- und analoger Reflexionswelt für die Entwicklung des individuellen Bürgerbewusstseins. Abschließend werden daraus Konsequenzen für politische Lernprozesse in der digitalisierten Welt abgeleitet.

Schlüsselwörter: politische Bildung, bürgerschaftliche Praxen, Bürgerbewusstsein, digitale Partizipation, Digitalisierung

\section{Citizenship Awareness, Political Learning and Participation in the Digitized World}

\section{Abstract}

Based on the importance of digital media and social networks in the everyday life of young people, this paper combines approaches from citizenship studies with learning theory approaches on citizen awareness. The relationship between digital experiences and its analogue reflections for the development of individual citizenship awareness is emphasized. Finally, consequences for political learning processes in the digitized world are deduced.

Keywords: citizenship education, acts of citizenship, digital acts, participation, citizenship awareness, digitalization

Die Digitalisierung stellt die politische Bildung vor neue Herausforderungen und prägt Lern- und Partizipationsprozesse in der Schule und darüber hinaus. Dennoch wird dieses Feld in der fachdidaktischen Forschung bis heute eher randständig behandelt. Im Fokus dieses Beitrags steht daher die grundlegende Frage danach, welche Prinzipien eine gelingende digital citizenship education (u. a. Kenner \& Lange, 2018, 
2020) kennzeichnen. Dafür gilt es, die Nutzungsgewohnheiten und Erfahrungen junger Menschen im digitalen Raum zu eruieren, die Besonderheiten digitaler Partizipationsprozesse zu beschreiben und die Bedeutung des Bürgerbewusstseins für eine subjektorientierte Lerntheorie im digitalen Zeitalter zu skizzieren.

Der folgende Beitrag widmet sich zunächst den Ergebnissen verschiedener Studien der quantitativen Sozialforschung zum Stellenwert digitaler Medien im Leben von Kindern, Jugendlichen und Erwachsenen. Dabei werden die veränderten Rahmenbedingungen für politische Lern- und Teilhabeprozesse im Zeitalter digitaler Transformationen herausgearbeitet. Diese Erkenntnisse werden im zweiten Teil mit dem Konzept der acts of citizenship (Isin, 2008) verknüpft. Dabei stellt sich die Frage, welche Formen des Handelns die digitale Welt kennzeichnen (Isin \& Ruppert, 2015). Anschließend wird thesenhaft der Einfluss der digitalen Erfahrungswelten auf die Entwicklung des Bürgerbewusstseins (Lange, 2008) des Individuums herausgearbeitet. Abschließend werden grundlegende Merkmale einer gelingenden digital citizenship education formuliert.

\section{Stellenwert digitaler Medien und sozialer Netzwerke - Empirische Befunde}

Basierend auf den repräsentativen Untersuchungen D21 DIGITAL INDEX 2018/2019, ARD/ZDF Onlinestudie 2019, KIM-Studie 2018, Shell Jugendstudie 2018, Engagiert aber allein und JIM-Studie 2018 wird im Folgenden der Stellenwert digitaler Medien und sozialer Netzwerke im Leben von Kindern, Jugendlichen und Erwachsenen untersucht. Im Fokus stehen dabei der Zugang zum Internet, das Nutzungsverhalten in Bezug auf digitale Medien und soziale Netzwerke sowie deren Auswirkungen auf das Verhältnis von Politik, Gesellschaft und Individuum.

Den Ergebnissen der vom Institut Kantar durchgeführten ARD/ZDF Onlinestudie 2019 zufolge nutzen derzeit rund 90 Prozent der Bevölkerung in Deutschland das Internet. Dieser Wert blieb im Vergleich zum Vorjahr konstant. Einen Anstieg um vier Punkte auf 71 Prozent verzeichnet die Zahl derer, die das Internet täglich nutzen. Bei den 14- bis 29-Jährigen gab mit 98 Prozent nahezu jede ${ }^{\star}$ Befragte an, täglich im Internet aktiv zu sein (ARD/ZDF-Forschungskommission, 2019, S. 3 f.). Auch die Ergebnisse des D21 DIGITAL INDEX 2018/2019 lassen darauf schließen, dass ein Großteil der Bevölkerung (84\%) das Internet nutzt. Auch hier ist die Generation unter 40 Jahren nahezu vollständig online (Initiative D21, 2019, S. 12). Dennoch kann den Zahlen dieser Untersuchungen zufolge davon ausgegangen werden, dass noch immer bis zu zehn Millionen Menschen in Deutschland das Internet weder beruflich noch privat nutzen (ebd.). Dabei ist zu unterscheiden, ob es sich um eine selbstbestimmte Nicht-Nutzung oder einen fremdbestimmten Ausschluss handelt 
(Kenner \& Lange, 2020). Selbstbestimmter Ausschluss wird dabei immer häufiger mit Sicherheitsbedenken begründet; fremdbestimmte Nicht-Nutzung ist beispielsweise auf ökonomische Faktoren zurückzuführen. Immerhin sieben Prozent der Befragten, die keinerlei Berührungspunkte mit dem Internet haben, gaben an, dass sie es sich finanziell nicht leisten könnten (Initiative D21, 2019, S. 18).

Bei der Nutzung digitaler Medien spielen vor allem soziale Netzwerke eine bedeutende Rolle. Das Internet wird in erster Linie als digitales Kommunikationsinstrument genutzt. Zwei von drei Befragten sind in den sozialen Medien aktiv, 56 Prozent nutzen WhatsApp, 41 Prozent haben einen Facebook-Account, und etwa jede ${ }^{\star}$ r Dritte schaut Videos auf der Plattform YouTube. Hervorzuheben ist, dass Menschen mit höherem Bildungsstand (78\%) deutlich häufiger soziale Medien nutzen als Menschen mit einem geringeren Bildungsstand (42\%) (ebd., S. 24).

Es bleibt zu konstatieren, dass bezugnehmend auf die Gesamtbevölkerung der überwiegende Teil der Menschen das Internet regelmäßig nutzt und dabei vor allem in sozialen Medien und Netzwerken aktiv ist. Insbesondere für die Generation unter 40 Jahren sind digitale Endgeräte aus dem Alltag kaum wegzudenken. Um die Frage zu beantworten, welchen Einfluss dieser digitale Medienkonsum auf Lernprozesse und die Entwicklung des Bürgerbewusstseins hat, ist es notwendig, einen genaueren Blick auf das Nutzungsverhalten und die Erfahrungen junger Menschen mit digitalen Medien zu werfen. Im folgenden Kapitel wird daher auf Grundlage empirischer Untersuchungen das Verhältnis von Kindern und Jugendlichen zu digitalen Medien nachgezeichnet.

Das Bürgerbewusstsein junger Menschen, die Gesamtheit aller mentalen Vorstellungen über die politisch-gesellschaftliche Wirklichkeit (Lange, 2008, S. 247), bildet sich in Lernprozessen, in Gesprächen, Begegnungen, im sozialen Umfeld, in der Familie, dem Freundeskreis, der Schule. Es dient der Orientierung in Politik und Gesellschaft. Um die Einflüsse digitaler Medien auf die Entwicklung des Bürgerbewusstseins verstehen zu können, ist es daher zunächst wichtig zu wissen, welchen Stellenwert persönliche Kontakte mit Freund*innen sowie der Familie in der realen Welt für junge Menschen heute noch einnehmen. Bei den Sechs- bis Dreizehnjährigen sind es noch immer knapp 90 Prozent der Befragten, die angeben, sich in ihrer Freizeit vorwiegend mit ihren Freund ${ }^{*}$ innen in der realen Welt zu treffen (MPFS, 2019, S. 11). Dies ergab die KIM-Studie 2018 des Medienpädagogischen Forschungsverbundes Südwest. In der ebenfalls im Jahr 2018 durchgeführten JIM-Studie wurden Jugendliche im Alter zwischen zwölf und 19 Jahren untersucht. Hier gaben nur noch knapp 71 Prozent der Befragten an, dass das Treffen mit Freund*innen die wichtigste Freizeitbeschäftigung sei (Feierabend, Rathgeb \& Reutter, 2018, S. 588). Wie wichtig es jungen Menschen ist, sich mit den engsten Bezugspersonen im Freundeskreis nicht nur im digitalen Raum, sondern auch im realen Umfeld auszutauschen, hat die 18. Shell Jugendstudie (Albert et al., 2019) 
ergeben. Die Autor*innen der Studie weisen darauf hin, dass hierbei Unterschiede je nach „der sozialen Herkunftsschicht“ (ebd., S. 157) erkennbar seien. „Acht von zehn Jugendlichen aus der oberen Schicht haben mit all ihren Freunden (auch) offline Kontakt. Bei Jugendlichen aus der unteren Schicht sind es lediglich sechs von zehn." (Ebd., S. 157 f.) Trotz der mit steigendem Alter sinkenden Wertschätzung des persönlichen Treffens mit Freundinnen belegen alle drei Untersuchungen, dass die Kommunikation mit Familie und Freundeskreis in der analogen, der realen Welt noch immer einen besonders hohen Stellenwert hat, denn es ist nach wie vor die wichtigste Freizeitbeschäftigung.

Doch auch in der digitalen Welt bewegen sich Kinder und Jugendliche, laut einer Studie der Vodafone Stiftung (2018), in sozialen Netzwerken hauptsächlich, um sich mit anderen Menschen auszutauschen. Dafür nutzen sie vor allem ihr Smartphone. Schon zwei von fünf Kindern im Alter zwischen sechs und dreizehn Jahren haben ein Smartphone (MPFS, 2019, S. 28). Im Altersverlauf nimmt der persönliche Besitz deutlich zu. Bei den Jugendlichen zwischen zwölf und 19 Jahren hat mit 97 Prozent nahezu jede*r ein internetfähiges Mobiltelefon (Feierabend et al., 2018, S. 587). Im Internet sind die Jugendlichen nach Selbsteinschätzung täglich durchschnittlich 3,7 Stunden (Albert et al., 2019, S. 30). Die Autor ${ }^{\star}$ innen der 18. Shell Jugendstudie kommen zu dem Schluss, dass es für alle Jugendlichen Normalität sei, viel Zeit im Internet zu verbringen (ebd.).

Doch wofür nutzen sie digitale Medien neben der Kommunikation in sozialen Netzwerken? Bei Kindern steht an erster Stelle der regelmäßig ausgeübten Tätigkeiten im Netz die Recherche über Suchmaschinen (MPFS, 2019, S. 32 f.). Auch für Jugendliche ist das Internet „keineswegs ein reines Unterhaltungsmedium“ (Albert et al., 2019, S. 30).

„An erster Stelle steht für sie Kommunikation: $96 \%$ sind mindestens einmal täglich in den sozialen Medien (Messengerdienste oder soziale Netzwerke) unterwegs. Zwar gehen $76 \%$ mindestens einmal am Tag aus Unterhaltungszwecken online (sei es für Musik, Videostreaming, Gamen oder Ansehen von Beiträgen von Personen, denen sie folgen), aber $71 \%$ suchen auch mindestens einmal täglich nach Informationen (allgemeiner Art, für Schule, Ausbildung oder Beruf oder über Politik und Gesellschaft). Deutlich seltener nutzen sie das Internet zur Selbstinszenierung, nur $12 \%$ stellen mindestens einmal täglich Fotos, Videos, Musik oder Blogbeiträge ins Netz“ (ebd., S. 30).

Für die politische Bildung sind in diesem Kontext auch die Ergebnisse der Onlinestudie der Vodafone Stiftung von Bedeutung. Sie lassen den Schluss zu, dass junge Menschen soziale Medien und Netzwerke auch als Chance für den Austausch, zumindest aber für die Auseinandersetzung mit der Politik begreifen. 
„Immerhin ein Viertel der Jugendlichen und junge [sic!] Erwachsenen folgt Politikerinnen und Politikern in den sozialen Medien. Mehr als jeder Dritte kann sich zudem vorstellen, politische Entscheidungsträger bei Fragen über soziale Medien direkt anzusprechen." (Vodafone Stiftung, 2018, S. 7)

Vor allem Kinder und Jugendliche informieren sich über gesellschaftliche Fragen und politische Zusammenhänge online (Albert et al., 2019, S. $287 \mathrm{ff}$.). Wenngleich das Vertrauen der jungen Menschen in klassische Medien wie die Tagesschau (84\%) und regionale Tageszeitungen (77\%) besonders hoch ist (Feierabend et al., 2018, S. 591), nutzen sie, um sich über gesellschaftspolitische Themen $\mathrm{zu}$ informieren, vor allem digitale Medien (Albert et al., 2019, S. 33). Dabei nutzen Befragte mit höherem Bildungsstand vorwiegend Online-Medien, um sich zu informieren; junge Menschen mit geringerem Bildungsstand bevorzugen soziale Netzwerke als Quelle. Interessant dabei ist, dass „nicht einmal jeder Dritte (29 Prozent) der Befragten Informationen und Nachrichten, die er über soziale Medien erhält, für ausgewogen" (Vodafone Stiftung, 2018, S. 13) erachtet. Dies ist nicht zuletzt darauf zurückzuführen, dass junge Menschen regelmäßig im Netz mit Falschinformationen und Hassnachrichten konfrontiert werden. „Rund zwei Drittel der jungen Menschen (66 Prozent) in Deutschland sehen durch Fake News den gesellschaftlichen Zusammenhalt gefährdet." (Ebd., S. 10) Die Befragten geben darüber hinaus an, dass die Schule sie auf den Umgang mit Falschnachrichten und Hasskommentaren nicht adäquat vorbereite. Bei Unsicherheit oder offenen Fragen zu Social Media suchen Jugendliche eher im Internet als in der Schule Rat (ebd., S. 13).

Wie lassen sich Handlungsoptionen basierend auf dem Nutzungsverhalten und den hier dargestellten Erfahrungen junger Menschen mit digitalen Medien und in sozialen Netzwerken kategorisieren? Dieser Frage wird im folgenden Kapitel nachgegangen.

\section{Being digital citizens - zur Bedeutung von Digitalisierung in der politischen Bildung}

Die Zahlen der im vorangegangenen Kapitel vorgestellten empirischen Untersuchungen offenbaren die enge Verzahnung von digitalen und sozialen Medien mit dem Leben von Kindern, Jugendlichen und Erwachsenen. Die digitale Welt ist interdependent mit dem Individuum, der Gesellschaft und der Politik verbunden. Die neuen technischen Möglichkeiten sind ein Produkt gesellschaftlicher Entwicklung und können zugleich ihr Antrieb sein. Digitale Medien schaffen eine erweiterte soziale und politische Realität. Menschen können durch die Teilnahme an digitalen Prozessen aktiv mitwirken und Verantwortung übernehmen. Dabei bezieht das nicht nur direktdemokratische Formate wie Online-Petitionen ein. Auch deliberative Teilhabe über digitale Bürgerversammlungen, repräsentative Beteiligung 
Bürgerbewusstsein, politisches Lernen und Partizipation im digitalen Zeitalter |

über den direkten Austausch beispielsweise mit den Wahlkreisabgeordneten über soziale Netzwerke und demonstrative Partizipation in Form von digitalem zivilen Ungehorsam sind möglich (Kersting, 2014, S. 60-79; Mülling, 2018).

Digitale Medien können auch dazu dienen, acts of citizenship (Isin, 2008) zu realisieren. Verstanden werden diese politischen Akte nicht oder nicht nur als Partizipation in den konventionellen und verfassten Strukturen. Engin F. Isin und Greg M. Nielsen, Politikwissenschaftler und renommierte Forscher im Feld der Citizenship Studies, definieren diese acts of citizenship wie folgt:

"They disrupt habitus, create new possibilities, claim rights and impose obligations in emotionally charged tones; pose their claims in enduring and creative expressions; and, most of all, are the actual moments that shift established practices, status and order. Acts of citizenship should be understood in themselves as unique and distinct from citizenship practices in the sense they are also actively answerable events, whereas the latter are institutionally accumulated processes." (Isin \& Nielsen, 2008, S. 10)

In dem Beitrag „Theorizing Acts of Citizenship“ beschreibt Isin welches CitizenshipVerständnis seiner Theorie zugrunde liegt. Er betont, dass die kritischen Citizenship Studies der letzten zwei Jahrzehnte offenbarten, dass Citizenship mehr ist als der Status in Form von Staatbürgerschaft. Citizenship impliziert für Isin auch bürgerschaftliche Praxen der Teilhabe, die sich von diesem engen Bürgerschaftsverständnis lösen. „[W]hat is important is not only that citizenship is a legal status but that it also involves practice of making citizens - social, political, cultural and symbolic." (Isin, 2008, S. 17)

Für die politische Bildung sind Bürgerschaft und Bürgerbewusstsein als Ausgangspunkt von Lernprozessen zusammenzudenken. Die Überlegungen von Isin und Nielsen gilt es dafür aus demokratietheoretischer Sicht um drei konzeptionelle $\mathrm{Zu}$ gänge zum Citizenship-Begriff zu ergänzen. Im liberalen Grundverständnis bezeichnet Citizenship eine Rechtsbeziehung zwischen dem Individuum und dem Staat. Die Staatsbürger*innen verfügen über spezifische Rechte (bspw. das Wahlrecht), die sie von der restlichen Bevölkerung unterscheiden. Politische Bildung will Menschen dazu befähigen, ihre Rolle im demokratischen Staat mündig auszuüben. Im didaktischen Zentrum steht das Prinzip der Interessenorientierung. Schüler ${ }^{\star}$ innen werden befähigt, ihre eigene Interessenlage zu erkennen, zu reflektieren und in die Gesellschaft einzuspeisen. Als geeignete Partizipationsform dieses liberalen Citizenship-Verständnisses erscheint die Repräsentation, sei es über Verbände, Vereine oder ein repräsentatives Wahlsystem. Ein wichtiges Bildungsziel ist dabei das Verständnis für die Institutionen und Verfahren des Staates (Hoskins, 2013, S. 26-28).

Im zweiten Grundverständnis wird Citizenship als soziale Praxis verstanden. Daraus resultiert eine politische Bildung, in der Citizenship als ein kollektives Unterfangen 
verstanden wird, das im zivilgesellschaftlichen Engagement der Menschen zum Ausdruck kommt. Dieses - eher republikanische - Citizenship-Verständnis zielt auf die direkte und kollektive Gestaltung der Gesellschaft durch ihre Mitglieder, unabhängig von ihrem rechtlichen Status als Bürger*innen. Citizenship ist dann Ausdruck einer selbstbewussten Zivilgesellschaft, die sich ihr Partizipationsrecht und ihre soziale Verantwortung nicht vom Staat übertragen lässt, sondern seit jeher beansprucht. Die Gemeinwohlorientierung schiebt sich vor die individuellen Interessen der Bürger ${ }^{\star}$ innen. Das Ziel ist bei diesem Verständnis von Citizenship die Befähigung zur Gestaltung der öffentlichen Belange (ebd., S. $28 \mathrm{f}$.).

Die Vertreter*innen der dritten großen Richtung, die auch an das CitizenshipVerständnis von Isin (2008) anschließt, begreifen Citizenship als eine kritische Haltung gegenüber der Gesellschaft. Die Partizipationsform stellt der Konflikt dar. Ein wesentliches Bildungsziel dieser ist es, die gesellschaftlichen Bedingtheiten und die sozialen Voraussetzungen $\mathrm{zu}$ erkennen und $\mathrm{zu}$ hinterfragen, die ungleich oder undemokratisch sind. Im Kern geht es darum, dass dem Subjekt durch politische Bildung Emanzipationsmöglichkeiten eröffnet werden sollen. Demokratiebildung zielt dann auf die Hinterfragung von Macht- und Herrschaftsstrukturen sowie auf Gesellschaftsveränderung nach den Prinzipien der Aufklärung und der Emanzipation. Citizenship in dieser Perspektive stellt letztlich auch immer eine Positionierung in hinterfragbaren gesellschaftlichen Strukturen dar (Hoskins, 2013, S. 29f.).

Für die digitale Welt ist vor allem der emanzipatorische Ansatz eines kritischen Citizenship-Verständnisses von besonderer Bedeutung. Das Hinterfragen von Machtund Herrschaftsstrukturen ist nicht nur für die analoge Welt eine relevante Fähigkeit, sondern ermöglicht auch im digitalen Raum, unterschiedliche Formate der Teilhabe zu kennen und ihre Bedingungen zu verstehen. Engin F. Isin und Evelyn Ruppert (2015) bezeichnen diese digitalen Teilhabemöglichkeiten als digital acts und haben diese in den folgenden drei Kategorien zusammengefasst: callings, closings und openings.

Als callings bezeichnen Isin und Ruppert die Aufforderung, Meinungen, Werte und Daten mit einer wachsenden Anzahl von Nutzer*innen zu teilen. Darunter subsumieren sie nicht nur das Handeln (participating), sondern auch das Vernetzen (connecting) und das Teilen (sharing) (ebd., S. 79). Dadurch hinterlassen die Menschen einen digitalen Fußabdruck. Fußabdruck steht symbolisch sowohl für das Hinterlassen von Spuren, die nachverfolgt werden können (Überwachung, fehlender Schutz der Privatsphäre), als auch für den historisch wirksamen Fußabdruck, für die Reichweite und den Einfluss, den das Wirken des Individuums im digitalen Raum erzeugen kann. Aktuell zeigt sich dies an der Vielzahl von Accounts der mittlerweile mehreren hundert Fridays-for-Future-Gruppen und vor allem an den Accounts ihrer Sprecher*innen. Wie auf callings reagiert wird und welche Rolle der individuelle digi- 
Bürgerbewusstsein, politisches Lernen und Partizipation im digitalen Zeitalter |

tale Fußabdruck in (sozialen) Netzwerken einnimmt, beeinflusst das individuelle politische Bewusstsein.

Menschen, die auf callings reagieren und digital handeln, sich vernetzen und etwas im Netz teilen, werden mit zwei Phänomenen konfrontiert, die Isin und Ruppert (2015) als closings und openings bezeichnen. Closings beziehen sich auf Vorschriften, Regelungen, systemische Bedingungen wie Filter oder Algorithmen, die eigene Aktionen und die verfügbaren Informationen einschränken (ebd., S. 107). Viele Nutzer ${ }^{\star}$ innen ordnen sich diesen Mechanismen meist unbewusst unter. Dies hängt auch damit zusammen, dass sie solche Phänomene nicht erkennen. $\mathrm{Zu}$ diesem Ergebnis kommt auch die Online-Studie der Vodafone Stiftung. Jede ${ }^{\star} r$ dritte Befragte gab an, nicht sicher zu sein, ob sie bzw. er Falschnachrichten identifizieren könnte (Vodafone Stiftung, 2018, S. 25). Die fehlende Kompetenz, Falschnachrichten zu entlarven oder die eigene Rolle in Filterblasen und Echokammern zu erkennen, kann nachhaltig Einfluss auf die Entwicklung des Bürgerbewusstseins nehmen. Die Gefahr besteht darin, dass die Auseinandersetzung mit anderen Positionen nicht mehr als fruchtbar, sondern als störend wahrgenommen wird (ausführlich dazu in Kap.4).

Im Gegensatz zu den closings beschreiben Isin und Ruppert die sogenannten openings als Möglichkeiten, anders zu denken, zu sprechen und $\mathrm{zu}$ handeln, indem man sich Konventionen widersetzt und neue Ideen entwickelt (Isin \& Ruppert, 2015, S. 64). Beispielhaft werden die Phänomene witnessing (bezeugen; bspw. whistleblowing - enthüllen/aufdecken), hacking und commoning beschrieben (ebd., S. $131 \mathrm{ff}$;; hierzu auch: Mülling, 2018). Mit commoning sind u. a. die vielfältigen Formen freier Software gemeint, die für alle Menschen zugänglich sind und von jedem auch weiterentwickelt werden können.

Darüber und durch andere digitale Formate der Beteiligung können Menschen Einfluss auf realpolitische Entscheidungsprozesse nehmen. Doch welche Kompetenzen sind erforderlich, um sich in einer digital erweiterten Demokratie autonom bewegen zu können? Was müssen Bürger*innen tun, um verantwortungsbewusst auf callings $\mathrm{zu}$ reagieren, kritisch-reflektiert mit closings umzugehen und selbstbestimmt openings zu nutzen? Für die Beantwortung dieser Fragen bedarf es breit angelegter empirischer Untersuchungen. Fest steht allerdings:

„Nicht anders als bei den früheren Innovationen gilt [...], dass digitale Medien nicht im sozialen Vakuum operieren. Das Netz ist ein Medium, dessen Wirkung von den Akteurinnen und Akteuren und ihren Nutzungsmotiven sowie von den von ihnen verbreiteten Botschaften abhängt." (Kneuer, 2017, S. 50)

Es hängt aber nicht nur das Netz von den Nutzer*innen ab. Das Netz, die Netzgemeinschaft, soziale Netzwerke, das Nutzungsverhalten digitaler Medien und Algorithmen verändern auch die Nutzer*innen. Es ist davon auszugehen, dass das Wirken 
in digitalen Sphären das Bürgerbewusstsein des Individuums und die Bereitschaft, eigene Vorstellungen kritisch zu hinterfragen, beeinflusst. Dies gilt es, auch für Lernprozesse in der Schule zu berücksichtigen. Im Folgenden wird daher das Konzept des Bürgerbewusstseins als Grundlage für eine subjektorientierte Lerntheorie unter Berücksichtigung des Einflusses digitaler Medien und sozialer Netzwerke vorgestellt.

\section{Das Bürgerbewusstsein in digitalen Bildungs- und Partizipationsprozessen}

Jeder Mensch baut mentale Vorstellungen auf, durch die er sich die politisch-gesellschaftliche Wirklichkeit sinnhaft macht. Die Gesamtheit dieser Vorstellungen lässt sich als Bürgerbewusstsein begreifen. Zum einen orientiert sich das Individuum mittels dieser Sinnbildungen in Politik, Wirtschaft und Gesellschaft. Zugleich stellen sie die kognitive Grundlage dar, um diese Phänomene zu beurteilen und zu kritisieren. Und schließlich produziert das Bürgerbewusstsein den Sinn, der es dem Menschen ermöglicht, vorgefundene Phänomene handelnd - also durch „subjektiv sinnhaftes Tun“ - zu beeinflussen.

Die Sinnbildungen des Bürgerbewusstseins im digitalen Raum sind didaktisch deshalb relevant, da sie Lernprozesse beeinflussen und sich in diesen verändern. Im Bürgerbewusstsein verschafft sich der Mensch ein Verständnis der politischen Dimension und der gesellschaftlichen Struktur des digitalen Raums. Es ist ein Lernprozess des doing digital citizenship. Die Sinnbildungen des Bürgerbewusstseins befähigen den Menschen, „bewußt zur Welt Stellung zu nehmen und ihr einen Sinn zu verleihen“ (Weber, 1985, S. 180). Sie ermöglichen es, „daß wir im Leben bestimmte Erscheinungen des menschlichen Zusammenseins aus ihm heraus beurteilen, zu ihnen als bedeutsam (positiv oder negativ) Stellung nehmen“ (ebd., S. 180 f.) können. Im Bürgerbewusstsein macht sich der Mensch einen „Ausschnitt aus der sinnlosen Unendlichkeit des Weltgeschehens“ (ebd., S. 180) politisch sinnhaft.

Ein großer Teil dieser zunächst sinnlos erscheinenden Unendlichkeit des Weltgeschehens spielt sich heute im Word Wide Web ab. Die politische Bedeutung des digitalen Raums ist nicht offenbar, sondern wird durch Bedeutungszuschreibungen und Sinnbildungen erst geschaffen. Die Vorstellungen über das Politische im digitalen Raum werden durch subjektive Plausibilisierungen des dort Erlebten hergestellt. Politische Lernprozesse finden demnach längst nicht mehr nur in formalen Bildungskontexten statt. Vielmehr finden sie - und damit auch die Lernprozesse des Bürgerbewusstseins - im politischen Alltag von Jugendlichen statt. Dieser Alltag erstreckt sich, wie die Ergebnisse der oben zitierten Studien belegen, zunehmend auf die digitalen und die sozialen Medien. Hier wird das Bedürfnis nach Plausibilisierung, nach Ausgewogenheit zwischen subjektiven Vorstellungen über 
gesellschaftliche Zusammenhänge und dem tatsächlichen Umwelterleben teilweise künstlich erzeugt bzw. technisch konstruiert. Vor allem die Global Player der Internetbranche, wie Google mit seinem Video-Netzwerk YouTube und Facebook mit seinen Netzwerken und Messenger-Diensten wie WhatsApp und Instagram, nutzen Algorithmen, um den Nutzer*innen eine Wohlfühlzone zu schaffen. Dafür stellen sie auf die Vorstellungswelten des Individuums zugeschnittene Informationen, Posts, Videos und Bilder zur Verfügung. Junge Menschen zu befähigen, sich dessen bewusst $\mathrm{zu}$ werden, ist eine zentrale Herausforderung der digital citizenship education.

Menschen erfahren aber im digitalen Raum nicht nur dessen Grenzen, die Isin und Ruppert (2015) als closings beschreiben. Dem „digitalen Citoyen“ (Thimm, 2016) öffnen sich vielmehr neue und andere Partizipationsräume. Der digitale politische Raum ist nicht nur eine augmented democracy; vielmehr werden in ihm zentrale Konzepte des Politischen - wie bspw. Öffentlichkeit, Willensbildung, Macht(kontrolle) - neu verhandelt (Kenner \& Lange, 2020).

Im Internet wird Politik gelernt, ohne dass sie gelehrt werden müsste. Fachdidaktisch interessant sind die mentalen Modelle, welche die politikrelevanten Gegenstände, Strukturen und Prozesse des World Wide Web subjektiv verständlich machen. Das Bürgerbewusstsein dient der Koordination in der digitalen politischen Wirklichkeit. Es muss durch politische Bildung aktiviert werden, um Kritikfähigkeit und Mündigkeit im digitalen Raum zu fördern. Doch wie bildet sich das Bürgerbewusstsein und wie lässt sich der Vorstellungswandel modellhaft darstellen und erklären?

Im Folgenden wird unter Bezug auf lernpsychologische und erziehungsphilosophische Ansätze (vgl. Piaget, 1992; Dewey, 1993) ein Lernmodell vorgeschlagen, das Lernenden als Motiv unterstellt, eine Kongruenz zwischen ihrem Bürgerbewusstsein und ihrem Erleben der politischen Wirklichkeit herstellen zu wollen. Dieses Kongruenzstreben ist nicht als ein Zustand, sondern als ein Prozess zu denken. Es beschreibt die Richtung, in die sich die andauernd mobilen Vorstellungswelten orientieren. Das Bürgerbewusstsein befindet sich in einem fortwährenden und unabgeschlossenen Prozess des politischen Lernens.

Der Ausgangspunkt ist ein Erleben von politischer Wirklichkeit, das mit den vorhandenen Bewusstseinsstrukturen nur unbefriedigend erklärt werden kann. Sobald der Mensch seine Aufmerksamkeit auf ein diskrepantes politisches Phänomen richtet, kommen seine politischen Vorstellungswelten in Unruhe, und er beginnt $\mathrm{zu}$ lernen. Diese Aufmerksamkeit ist wesentlich. Denn der Mensch hat täglich eine Vielzahl bewusstseinsdiskrepanter Erlebnisse und lässt dennoch viele der politischen Lerngelegenheiten aus. Das gilt für die analoge wie für die digitale Lebenswelt. Warum darf ich kostenlos Suchmaschinen benutzen? Wieso bekomme ich Werbematerial einer bestimmten politischen Partei? Weiß der Internetkonzern, auf wel- 
che politischen Veranstaltungen ich gehe? Wie kann ich Menschen für eine konkrete Aktion gewinnen? Die Lebenswelt Internet ist politisch äußerst relevant und führt zu unzähligen Fragen, die eine Diskrepanz zwischen den subjektiven Vorstellungen und dem realen Umwelterleben erzeugen könnten. Aber ob tatsächlich politisch gelernt wird, hängt auch davon ab, ob Diskrepanz bewusst wird. Das Erleben wird erst lernwirksam, wenn es reflektiert und zu einer Erfahrung (im Sinne John Deweys) wird. Der Erfahrungsbegriff betont die Diskrepanz, die Lernende zwischen ihrem Bürgerbewusstsein und ihrem Umwelterleben subjektiv wahrnehmen. Damit beginnt ein Prozess, der auf potenzielle Wiederherstellung der Kongruenz von politischen Vorstellungswelten und dem Umwelterleben abzielt. Die Aufmerksamkeit der Lernenden auf diese Diskrepanz zu lenken, muss das Ziel von digital citizenship education sein.

Politische Bildung basiert nicht auf der Übernahme von Fachwissen beziehungsweise auf dem Austausch alltäglicher durch wissenschaftlich fundierte Konzepte. Sie unterliegt den Formen und der Dynamik der bestehenden fachlichen Vorstellungswelten der Lernenden. Der Lernprozess findet seinen Ausgangspunkt in dem vorhandenen Bürgerbewusstsein. Zu den Aufgaben der Didaktik der politischen Bildung zählt es, die fachlichen Voraussetzungen in ihrer Bedeutung für den Lernprozess zu reflektieren. Denn als Impulse für Lernprozesse kommen nur Erlebnisse in Betracht, die mit dem Bürgerbewusstsein der Lernenden in Verbindung gebracht werden können. Andernfalls bewirkt das Erlebnis mit der politisch-gesellschaftlichen Wirklichkeit keine Erfahrung. Es bleibt lernunwirksam.

Trotz Filterblasen und Echokammern - die Vorstellungswelten eher beruhigen als mobilisieren - werden heute wesentliche Diskrepanzerfahrungen im Internet gemacht. Es können gesellschaftliche Perspektiven, politische Einstellungen und soziale Orientierungen kennengelernt werden, die im analogen Raum unzugänglich geblieben wären. Insofern bietet das Internet ein enormes Reservoir für politisches Lernen.

Politische Bildung im digitalen Raum sollte reflektieren, wie Lernende mit ihren fachlichen Vorstellungen am Lernprozess beteiligt sind und welche neuen Erfahrungen sie dabei machen. Wer eine politische Erfahrung macht, erfährt dies im Bürgerbewusstsein als eine mentale Dissonanz. Die irritierende Erfahrung wird in einem fragenden und problembewältigenden Vorgang angeeignet, wodurch die Unstimmigkeit tendenziell beseitigt wird. Politische Bildung sollte deshalb reflektieren, auf welchen individuellen Wegen Lerngegenstände angeeignet werden. Es interessieren die Denkerfahrungen und Problembewältigungen, die den Verinnerlichungsprozess leiten. Hierbei muss die digitale Lebenswelt mir der analogen Reflexionswelt didaktisch in Beziehung gesetzt werden. 


\section{Konsequenzen für politische Lernprozesse in der digitalisierten Welt}

Eine Didaktik des politischen Lernens im digitalen Zeitalter fokussiert die differenzierte Auseinandersetzung mit Möglichkeiten, Potenzialen und Chancen, aber auch Gefahren und Risiken der Digitalisierung für Bildungs- und Teilhabeprozesse. Die eingangs zitierten repräsentativen Untersuchungen zeigen, dass das Internet, das Smartphone und soziale Netzwerke selbstverständlicher Bestandteil im Alltag junger Menschen geworden sind. Sie ermöglichen vielfältige Lern- und Partizipationsanlässe, die es auf Grundlage einer subjektorientierten Lerntheorie und unter Berücksichtigung des Bürgerbewusstseins zu fördern gilt. So kann politische Bildung auch eine Auseinandersetzung mit Macht- und Herrschaftsverhältnissen und deren Verschiebungen durch die Digitalisierung ermöglichen. Besonders wichtig ist es, die Nutzungsgewohnheiten junger Menschen zu kennen und ihre Erfahrungen im Netz mit ihnen gemeinsam zu reflektieren. Für die Kinder und Jugendlichen sind digitale Medien und soziale Netzwerke selbstverständlicher Teil ihres Lebens. Sie prägen ihren Alltag und damit auch ihre alltäglichen Lernerfahrungen. Eine subjektorientierte Didaktik, die nicht nur fachwissenschaftliche Konzepte vermitteln, sondern an die lebensweltlichen, subjektiven Vorstellungen der Schüler ${ }^{\star}$ innen anschließen will, muss sich mit den Herausforderungen der digitalisierten Sphären befassen. Um auf die neuen Herausforderungen des digitalen Zeitalters adäquat reagieren zu können und eine gelingende digital citizenship education zu ermöglichen, bedarf es demnach:

- einer Auseinandersetzung mit Nutzungsgewohnheiten und Erfahrungen junger Menschen im digitalen Raum,

- des Aufgreifens und Ergründens subjektiver Vorstellungswelten (Bürgerbewusstsein), auch über den digitalen Raum,

- der Entwicklung technischer Fähigkeiten als Voraussetzung für den reflektierten Umgang mit digitalen Medien,

- digitaler Orientierungskompetenzen, um sich in den neuen politischen Sphären zu bewegen, Fake News zu identifizieren und sich der Wirkung von Filterblasen und Algorithmen bewusst zu sein,

- der Fähigkeit, neue Macht- und Herrschaftsverhältnisse im digitalen Raum kritisch zu reflektieren,

- digitaler Partizipationsfähigkeiten, um eigene Interessen im Internet zu artikulieren und zu vertreten sowie für die Wahrung der Rechte anderer (Grund- und Menschenrechte) eintreten zu können.

Wenngleich auch andere mediale Innovationen in der Geschichte die Kommunikation und politische Lernprozesse nachhaltig beeinflusst haben, so sind vor allem die Geschwindigkeit der technologischen Entwicklungen und die damit verbundenen Dynamiken im digitalen Raum beispiellos (vgl. Kneuer, 2017). Junge Menschen nutzen diese neu entstehenden Räume, um Erfahrungen zu sammeln, um zu lernen und sich selbst zu bilden. Sie finden in diesen digitalen Sphären auch neue Hand- 
lungsoptionen, die wiederum neue Lernanlässe schaffen. Einer modernen Didaktik der politischen Bildung gelingt es, diesen Prozess zu begleiten, wenn sie das Subjekt, das Bürgerbewusstsein mit seinen mobilen Vorstellungswelten in den Fokus didaktischer Arbeit rückt.

\section{Literatur und Internetquellen}

Albert, A., Hurrelmann, K., Quenzel, G., Schneekluth, U., Leven, I., Utzmann, H., \& Wolfert, S. (2019). Jugend 2019 - 18. Shell Jugendstudie: Eine Generation meldet sich zu Wort. Weinheim \& Basel: Beltz. https://doi.org/10.3224/diskurs.v14i4.06

ARD/ZDF-Forschungskommission (2019). Ergebnisse der ARD/ZDF Onlinestudie 2019. Zugriff am 02.01.2020. Verfügbar unter: http://www.ard-zdf-onlinestudie.de/files/2019/ Ergebnispraesentation_ARD_ZDF_Onlinestudie_PUBLIKATION_extern.pdf.

Dewey, J. (1993). Demokratie und Erziehung. Eine Einleitung in die philosophische Pädagogik. Hrsg. von J. Oelkers. Weinheim \& Basel: Beltz.

Feierabend, S., Rathgeb, T., \& Reutter, T. (2018). Jugend, Information, Medien. Ergebnisse der JIM-Studie 2018. Media Perspektiven, (12), 587-600.

Hoskins, B. (2013). What Does Democracy Need from Its Citizens? In M. Print \& D. Lange (Hrsg.), Civic Education and Competences for Engaging Citizens in Democracies (S. 2336). Rotterdam: Sense Publishers. https://doi.org/10.1007/978-94-6209-172-6_3

Initiative D21 (2019). D21 DIGITAL INDEX 2018/2019. Jährliches Lagebild zur Digitalen Gesellschaft. Berlin: Stoba-Druck. Zugriff am 02.01.2020. Verfügbar unter: https://ini tiatived21.de/publikationen/d21-digital-index-2018-2019/.

Isin, E. F. (2008). Theorizing Acts of Citizenship. In E. F. Isin \& G. M. Nielsen (Hrsg.), Acts of Citizenship (S. 15-43). London \& New York: Zed Books.

Isin, E. F., \& Nielsen, G. M. (2008). Introduction. In E. F. Isin \& G. M. Nielsen (Hrsg.), Acts of Citizenship (S. 11-13). London \& New York: Zed Books.

Isin, E. F., \& Ruppert, E. (2015). Being Digital Citizens. London \& New York: Rowman \& Littlefield.

Kenner, S., \& Lange, D. (2018). Digital Citizenship Education. Digitale Medienkompetenz als Aufgabe der Politischen Bildung. Informationen zur Politischen Bildung, 43 (Medien und politische Kommunikation), 13-18.

Kenner, S., \& Lange, D. (2020). Digitalisierung als Herausforderung für politische Bildung. Im Spannungsfeld von inklusiven Praxen und neuen Formen der Exklusion. In A. Albrecht, G. Bade, A. Eis, U. Jakubczyk \& B. Overwien (Hrsg.), Wann, wenn nicht jetzt? Politische Bildung in der Schule stärken (S. 233-248). Frankfurt a. M.: Wochenschau.

Kersting, N. (2014). Online Beteiligung - Elektronische Partizipation - Qualitätskriterien aus Sicht der Politik. In K. Voss (Hrsg.), Internet und Partizipation. Bottom-up oder Top-down? Politische Beteiligungsmöglichkeiten im Internet (S. 53-87). Wiesbaden: Springer VS. https://doi.org/10.1007/978-3-658-01028-7_3

Kneuer, M. (2017). Politische Kommunikation und digitale Medien in der Demokratie. In H. Gapski, M. Oberle \& W. Staufer (Hrsg.), Medienkompetenz. Herausforderung für Politik, politische Bildung und Medienbildung (S. 43-52). Bonn: Bundeszentrale für politische Bildung.

Lange, D. (2008). Kernkonzepte des Bürgerbewusstseins. Grundzüge einer Lerntheorie der politischen Bildung. In G. Weißeno (Hrsg.), Politikkompetenz. Was Unterricht zu leisten hat (S. 245-258). Bonn: Bundeszentrale für politische Bildung.

MPFS (Medienpädagogischer Forschungsverbund Südwest) (2019). KIM-Studie. Kindheit, Internet, Medien. Basisuntersuchung zum Medienumgang 6- bis 13-Jähriger. Zugriff am 
Bürgerbewusstsein, politisches Lernen und Partizipation im digitalen Zeitalter |

02.02.2020. Verfügbar unter: https://www.mpfs.de/fileadmin/files/Studien/KIM/2018/ KIM-Studie_2018_web.pdf.

Mülling, E. (2018). Big Data und der zivile Ungehorsam. Wiesbaden: Springer VS. https:// doi.org/10.1007/978-3-658-24159-9

Piaget, J. (1992). Das Erwachen der Intelligenz beim Kinde. Stuttgart: Klett-Cotta.

Thimm, C. (2016). Digitale Demokratie im Kreuzfeuer - Der mühsame Weg zum Digitalen Citoyen. In I. Pöttinger, R. Fries \& T. Kalwar (Hrsg.), Doing Politics - politisch agieren in der digitalen Gesellschaft (S. 51-68). München: kopaed.

Vodafone Stiftung Deutschland (2018). ENGAGIERT ABER ALLEIN. Wie sich junge Menschen durch die Online-Welt navigieren und welche Unterstützung sie dafür suchen. Zugriff am 02.01.2020. Verfügbar unter: https://www.vodafone-stiftung.de/wp-content/ uploads/2019/04/Vodafone_Stiftung_Engagiert_aber_allein_18_01.pdf.

Weber, M. (1985). Gesammelte Aufsätze zur Wissenschaftslehre (UTB für Wissenschaft; 6., erneut durchges. Aufl.). Tübingen: Mohr.

Steve Kenner, wissenschaftlicher Mitarbeiter am Institut für Didaktik der Demokratie (IDD) und Mitglied der Geschäftsführung des Center for Inclusive Citizenship (CINC), beides an der Leibniz Universität Hannover.

E-Mail: steve.kenner@idd.uni-hannover.de

Dirk Lange, Prof. Dr., lehrt Didaktik der Politischen Bildung an der Universität Wien und an der Leibniz Universität Hannover.

E-Mail: lange@idd.uni-hannover.de

Korrespondenzadresse: Leibniz Universität Hannover, Institut für Didaktik der Demokratie, Callinstr. 20, 30167 Hannover 